\title{
Wernicke Encephalopathy in a Child with Acute Lymphoblastic Leukemia with Atypical Neuroimaging Findings
}

\begin{abstract}
Wernicke encephalopathy (WE) is characterized by a clinical triad of ataxia, ophthalmoplegia, and altered mental state. It is said to be a rare entity in infants and children. We describe the case of a 2-year-old child with acute leukemia on chemotherapy who presented with acute encephalopathy. Blood parameters and cerebrospinal fluid analysis were normal, and magnetic resonance imaging of the brain revealed isolated cranial nerve nuclei involvement. There was remarkable improvement in sensorium following thiamine therapy. A high index of suspicion is necessary to diagnose WE in high-risk patients such as leukemia.
\end{abstract}

Keywords: Acute encephalopathy, acute lymphoblastic leukemia, Wernicke encephalopathy

\section{Introduction}

Wernicke encephalopathy (WE) is a medical emergency caused by thiamine deficiency. It is an acute neurological condition characterized by a clinical triad of ophthalmoparesis with nystagmus, ataxia, and confusion. Pediatric WE has been described in severe malnutrition, starvation, prolonged parenteral nutrition, and malignancies, following gastrointestinal surgeries and stem cell transplantation. ${ }^{[1,2]}$ Characteristic brain magnetic resonance imaging (MRI) findings include symmetric T2 hyperintensities in dorsal medial thalamus, mammillary bodies, periaqueductal gray matter, and tectal plate. Selective involvement of the cranial nerve nuclei, cerebellum, red nuclei, dentate nuclei, corpus callosum, cerebral cortex, and basal ganglia has been described in nonalcoholic WE patients. ${ }^{[3]}$ Here, we report a case of WE in a child with acute Lymphoblastic leukemia with atypical MRI findings.

\section{Case Report}

A previously healthy 2-year-old female child who had been diagnosed with acute lymphoblastic leukemia 5 months ago presented with excessive sleepiness, irritability, and incessant cry of 3-day

\footnotetext{
This is an open access journal, and articles are distributed under the terms of the Creative Commons Attribution-NonCommercialShareAlike 4.0 License, which allows others to remix, tweak, and build upon the work non-commercially, as long as appropriate credit is given and the new creations are licensed under the identical terms.
}

For reprints contact: WKHLRPMedknow_reprints@wolterskluwer.com duration. She is on maintenance phase chemotherapy according to the Indian childhood collaborative leukemia protocol and a case of standard risk under risk stratification. There was no history of fever, loose stools, vomiting, or seizures. On examination, the child was drowsy, arousable but irritable, was not interacting, and not communicating. Extraocular movements were full and pupils equal and reacting to light. She was moving all her limbs, however was not sitting or standing on her own. There was generalized hypotonia; deep tendon reflexes were normal with flexor plantars. There was no nystagmus, head nodding, or intention tremors, and there were no meningeal signs.

Leukemic infiltration of the central nervous system, central nervous system infections in an immunocompromised state, drug toxicity-chemical meningitis, leukoencephalopathy, and posterior reversible encephalopathy syndrome were considered as probable differentials, and investigations were planned.

Blood counts were normal; metabolic parameters including urea, creatinine, sugar, electrolytes, liver enzymes, and serum ammonia were normal. Cerebrospinal fluid (CSF) analysis was normal with normal biochemistry, gram stain, acid-fast bacillus stain, and fungal stain. There were no blasts in the CSF. Culture including fungal culture was sterile. CSF viral studies

\footnotetext{
How to cite this article: Cornelius LP, Paulraj AJ, Elango N. Wernicke encephalopathy in a child with acute lymphoblastic leukemia with atypical neuroimaging findings. Indian J Med Paediatr Oncol 2020;41:767-9.
}

\author{
Leema Pauline \\ Cornelius, \\ Asir Julin Paulraj, \\ Neeraj Elango \\ Department of Paediatric \\ Neurology, Institute of Child \\ Health and Hospital for \\ Children, Madras Medical \\ College, Chennai, Tamil Nadu, \\ India
}

Submitted: 21-Mar-2020

Revised: 14-May-2020

Accepted: 27-May-2020

Published: 29-Oct-2020

Address for correspondence:

Dr. Leema Pauline Cornelius, Department of Paediatric Neurology, Institute of Child Health and Hospital for Children, Egmore, Chennai, Tamil Nadu, India.

E-mail: leema.pauline@ rediffmail.com

Access this article online

Website: www.ijmpo.org

DOI: 10.4103/ijmpo.ijmpo_106_20 Quick Response Code:

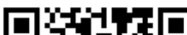


including Japanese encephalitis virus, herpes simplex, varicella, and enterovirus were negative. MRI of the brain showed symmetric high signal alterations in facial nerve nuclei with diffusion restriction [Figure 1a-c]. There was no involvement of the thalamus, mammillary bodies, periaqueductal gray matter, or tectum in the midbrain [Figure 1d-f].

Based on the clinical and imaging findings, WE was considered, and injection thiamine $100 \mathrm{mg}$ intravenously twice daily was given for 3 days and once daily for the next 4 days. A rapid improvement in the neurological status was noticed within $24 \mathrm{~h}$ of thiamine treatment. She became active, alert, communicating, and started walking and playing on the $4^{\text {th }}$ day.

\section{Discussion}

WE is characterized by a clinical triad of gait ataxia, eye signs (nystagmus and ophthalmoplegia), and global confusion as described by Carl Wernicke in 1881 . However, this classic triad is observed only in $21.4 \%$ of pediatric patients. ${ }^{[4]}$ The most constant clinical finding is mental status changes that comprise a state of confusion, disorientation, drowsiness, cognitive impairment with disturbance in memory, and coma. The child in the case above presented with lethargy and drowsiness only and did not have ataxia or ophthalmoparesis.

Several types of cancers such as acute lymphoblastic leukemia, acute mixed lineage leukemia, acute myeloid leukemia, germ-cell tumor, and osteosarcoma have been associated with WE. Factors including increased consumption of thiamine by rapidly growing cancer cells, chemotherapeutic agent interference with function of thiamine, poor intake, vomiting, and liver disease leading to decreased conversion of thiamine contribute to thiamine deficiency in these children. ${ }^{[5]}$

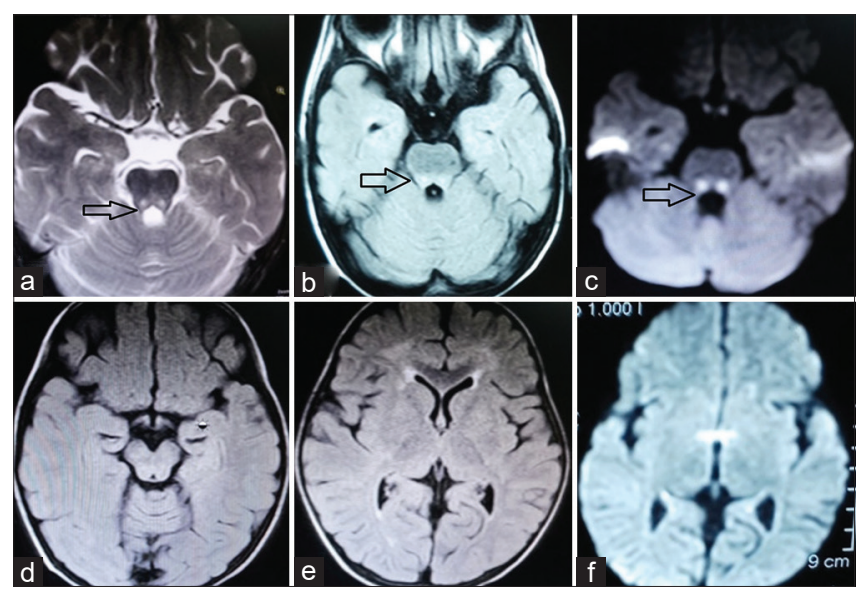

Figure 1: ( $a$ and $b$ ) Axial magnetic resonance images. T2-weighted, fluidattenuated inversion recovery sequences showing symmetric high-signal alterations in facial nerve nuclei in the pons. (c) Diffusion-weighted imaging showing diffusion restriction of facial nerve nuclei. (d-f) Axial fluid-attenuated inversion recovery and diffusion-weighted imaging images showing normal midbrain and thalami
Thiamine acts as a cofactor for various enzymes in the carbohydrate metabolism including transketolase, alphaketoglutarate dehydrogenase, pyruvate dehydrogenase, and branched-chain ketoacid dehydrogenase complex which play a key role in the regulation of glucose and energy metabolism in the brain. Thiamine deficiency is hypothesized to cause neuronal injury in the brain regions with high metabolic requirements. Oxidative stress, glutamate-mediated excitotoxicity, and inflammation are major contributors to regional neurodegeneration in WE. ${ }^{[6]}$

Brain MRI is the most sensitive technique and is required for all patients in whom WE is suspected. Typical imaging findings include bilateral, symmetrical high-signal intensities in the thalami, mammillary bodies, tectal plate, and periaqueductal area. The dorsal medulla, red nuclei, cranial nerve nuclei, cerebellum, callosum, and frontal and parietal cerebral cortex are less common sites of involvement. Selective cranial nerve nuclei involvement has been reported in WE. ${ }^{[3]}$ Liou et al. described a case of acute leukemia affected by WE with lesions in the olivary bodies, cranial nerve nuclei, and dentate nuclei in the absence of lesions in the typically affected areas. ${ }^{[7]}$ Our child showed the involvement of facial nerve nuclei in the pons alone.

The differential diagnosis of symmetric signal-intensity alterations of the dentate, cranial nerve nuclei, red nuclei, and splenium includes metronidazole-induced encephalopathy. ${ }^{[8]}$ However, metronidazole was not used in our case. The other differential to be considered is leukoencephalopathy due to methotrexate toxicity, but involvement of the centrum semiovale is the predominant imaging finding in methotrexate toxicity. Furthermore, rapid improvement in sensorium with thiamine supplementation clinches the diagnosis of WE. Leigh syndrome, a mitochondrial disorder with progressive neurodegeneration, can have similar imaging features - symmetrical T2 highsignal intensities in the brainstem, thalami, basal ganglia, and periaqueductal grey matter, but mammillary bodies are not involved.

WE is a medical emergency, and any therapeutic delay may result in permanent neurological damage or death. Empiric thiamine replacement should be initiated as soon as WE is suspected. There is no fixed guideline for pediatric patients. Hundred milligrams of daily thiamine replacement is routinely recommended. ${ }^{[9]}$ Medical literature suggests that pediatric patients at risk for thiamine deficiency be supplemented with $0.35-0.5 \mathrm{mg} / \mathrm{kg} /$ day of oral thiamine to prevent the development of encephalopathy. Children with malignancies are at high risk for the development of WE and could be given excessive thiamine. However, this practice remains controversial as a large amount of thiamine may promote tumor cell growth. It is difficult to make specific recommendations regarding the treatment of WE in children because of a paucity of the literature. ${ }^{[10]}$ 


\section{Conclusion}

Awareness of heterogeneity in clinical and imaging features is fundamental to recognize WE, especially in children. Thiamine therapy is warranted if any component of the WE triad is present in an appropriate clinical setting.

\section{Declaration of patient consent}

The authors certify that they have obtained all appropriate patient consent forms. In the form, the legal guardian has given consent for images and other clinical information to be reported in the journal. The guardian understands that name and initial will not be published and due efforts will be made to conceal the identity, but anonymity cannot be guaranteed.

\section{Financial support and sponsorship}

Nil.

\section{Conflicts of interest}

There are no conflicts of interest.

\section{References}

1. Kamaşak T, Kul S, Tuşat M, Ozgun N, Cansu A. A case of Wernicke encephalopathy developing after Ileal bypass surgery.
Pediatr Emerg Care 2018;34:e223-5.

2. Ghorbani A, Fatehi F, Soltanzadeh A, Hamidieh A, Vahabi Z. Wernicke's encephalopathy in a young girl suffering from acute myeloblastic leukemia. Iran J Pediatr 2012;22:574-5.

3. Zuccoli G, Motti L. Atypical Wernicke's encephalopathy showing lesions in the cranial nerve nuclei and cerebellum. J Neuroimaging 2008;18:194-7.

4. Vasconcelos MM, Silva KP, Vidal G, Silva AF, Domingues RC, Berditchevsky CR. Early diagnosis of pediatric Wernicke's encephalopathy. Pediatr Neurol 1999;20:289-94.

5. Lim YJ, Kim HJ, Lee YJ, Seol IJ, Lee YH. Clinical features of encephalopathy in children with cancer requiring cranial magnetic resonance imaging. Pediatr Neurol 2011;44:4338.

6. Hazell AS, Butterworth RF. Update of cell damage mechanisms in thiamine deficiency: Focus on oxidative stress, excitotoxicity and inflammation. Alcohol Alcohol 2009;44:141-7.

7. Liou KC, Kuo SF, Chen LA. Wernicke encephalopathy with atypical magnetic resonance imaging. Am J Emerg Med 2012;30:2086.e1-3.

8. Zuccoli G, Pipitone N, Cruz DS. Metronidazole-induced and Wernicke encephalopathy: Two different entities sharing the same metabolic pathway? Am J Neuroradiol 2008;29:E84.

9. Manzanares W, Hardy G. Thiamine supplementation in the critically ill. Curr Opin Clin Nutr Metab Care 2011;14:610-7.

10. Lallas M, Desai J. Wernicke encephalopathy in children and adolescents. World J Pediatr 2014;10:293-8. 